(1)

CrossMark

\title{
Will children reveal their secret? The coronavirus dilemma
}

\author{
To the Editor:
}

I was interested to read the recent editorial: "Will children reveal their secret? The coronavirus dilemma" [1]. While well substantiated, the assertion that increased angiotensin-converting enzyme 2 (ACE2) expression is protective in terms of coronavirus disease 2019 (COVID-19) outcome seems to conflict with a large body of literature on the topic. I would therefore like to briefly explore some of the arguments around this, with the hope of highlighting the main areas of conflict and inciting more research on this aspect of severe acute respiratory syndrome coronavirus 2 (SARS-CoV-2) pathophysiology.

ACE2 is an important component of the renin-angiotensin system (RAS), which acts as the receptor for SARS-CoV-2. Many authors argue that ACE2 is the underlying reason behind a number of the risk factors for severe COVID-19. FANG et al. [2] state that treatment of conditions such as hypertension and diabetes with ACE inhibitors (ACEI) and angiotensin-II receptor blockers (ARB) results in significant upregulation of ACE2, offering more ports of entry into cells by SARS-CoV-2, resulting in the worst outcomes reported in these patients. In a similar vein, LEUNG et al. [3] studied the expression of ACE2 via immunohistochemistry in human lung biopsies. They found significantly higher expression of ACE2 in smokers and COPD patients compared with healthy controls, and argue that this is why smoking status and COPD appear associated with negative COVID-19 outcomes [3]. Finally, a small study that measured the serum levels of ACE2 in patients with COVID-19 found that they were significantly higher $(\mathrm{p}<0.001)$ than healthy controls and levels closely correlated with lung injury and viral load [4].

Despite evidence that ACE2 is implicated in the pathology of COVID-19, we should not jump to conclusions about its exact role. Both the study of lung biopsies and the serum measurement of ACE2 had low sample sizes, at 27 and 20 including controls, respectively [2, 3]. Moreover, much of the epidemiological evidence could be confounded by the fact that many of the conditions that reportedly raise ACE2 are associated with age and obesity, which are also implicated in the negative outcomes of both SARS-CoV-2 infection and that of other respiratory viruses [5]. Equally, systematic reviews of the hypothesised effect of ACEI/ARB on COVID-19 outcomes have so far not found any significant effect [5, 6]. Much of the pathophysiology and epidemiology of SARS-CoV-2 is not yet understood, so it is unsurprising that definitive conclusions are yet to be drawn. Despite this, there are clear grounds to question the argument that ACE2 overexpression directly contributes to negative COVID-19 outcomes.

There is a growing body of literature that argues that ACE2 upregulation is a protective factor for SARS-CoV-2 outcomes. Models of acute respiratory distress syndrome (ARDS) in ACE2 knockout mice have shown that ACE2 confers a protective effect [7]. Additionally, injecting the SARS-CoV (from the 2002 outbreak) spike into mice that already have ARDS dramatically worsens their condition, a process that can then be ameliorated by blocking RAS [7]. Therefore, in mice it is possible that ARDS in SARS-CoV is mediated at least in part by ACE2 disruption and resulting RAS dysregulation. Moreover, in vitro reports suggest that SARS-CoV-2 actually downregulates ACE2 after using it for cellular entry, resulting in immune cell infiltration, RAS dysregulation and lung injury [5]. Meanwhile, the aforementioned editorial highlights rat models that illustrate a significant decrease in ACE2 expression with age, which, if also exhibited in humans, could form part of the reason that children are far less effected by COVID-19 than adults [1].

This hypothesis is also not without flaws. The murine models illustrate that a complete lack of ACE2 results in dysregulated RAS and more severe ARDS versus normal expression. However, the theoretical

@ERSpublications

A recent $E R J$ editorial cited ACE2 upregulation as a key reason for the better outcomes of COVID-19 seen in paediatric patients. However, there is significant conflicting literature on the topic, and many questions about ACE2 physiology still unanswered. https://bit.ly/2Skh2Lp

Cite this article as: Porter GJ. Will children reveal their secret? The coronavirus dilemma. Eur Respir J 2020; 55: 2001382 [https://doi.org/10.1183/13993003.01382-2020]. 
comparison in actuality is increased ACE2 expression versus normal or slightly decreased levels. Moreover, while rat models show a significant decrease in ACE2 expression with age, SCHOUTEN et al. [8] reported no significant differences in lung ACE2 activity between neonates, children, adults and older adults ( $>65$ years old), while FERNÁNDEZ-ATUCHA et al. [9] found significantly higher ACE2 activity in women $>55$ years of age, and no difference in men. There is also considerable evidence that ACEI/ARB therapy makes no difference in ACE2 expression, meaning arguments surrounding the lack of association between these medications and COVID-19 outcomes may be invalid, regardless of how they characterise the role of ACE2 [5].

Much of the literature on ACE2's role in COVID-19 is highly conflicting. I believe that this stems from the fact that basic mechanisms of ACE2 physiology, such as the effect of aging or ACEI/ARB usage on its expression, or how ACE2 levels change during COVID-19 infection, are still debated. Understanding the role of ACE2 could offer insight both into the prognosis of individual COVID-19 cases and therapeutic techniques $[1,4]$. Research in this area is therefore vital, particularly robust case-control studies of ACE2 expression and COVID outcomes and genomic studies of ACE2 expression at different ages and disease states [1].

\section{George James Porter}

Faculty of Medical Sciences, Newcastle University, Newcastle Upon Tyne, UK.

Correspondence: George James Porter, 27 Gaywood Street, London, SE16HG UK. E-mail: g.porter2@newcastle.ac.uk

Received: 24 April 2020 | Accepted: 27 April 2020

Conflict of interest: G.J. Porter has nothing to disclose.

\section{References}

1 Cristiani L, Mancino E, Matera L, et al. Will children reveal their secret? The coronavirus dilemma. Eur Respir J 2020; 55: 2000749.

2 Fang L, Karakiulakis G, Roth M. Are patients with hypertension and diabetes mellitus at increased risk for COVID-19 infection? Lancet Respir Med 2020; 8: PE21.

3 Leung JM, Yang CX, Tam A, et al. ACE-2 expression in the small airway epithelia of smokers and COPD patients: implications for COVID-19. Eur Respir J 2020; 55: 2000688.

4 Liu Y, Yang Y, Zhang C, et al. Clinical and biochemical indexes from 2019-nCoV infected patients linked to viral loads and lung injury. Sci China Life Sci 2020; 63: 364-374.

5 Vaduganathan $\mathrm{M}$, Vardeny $\mathrm{O}$, Michel $\mathrm{T}$, et al. Renin-angiotensin-aldosterone system inhibitors in patients with COVID-19. N Engl J Med 2020; 382: 1653-1659.

6 Rico-Mesa JS, White A, Anderson AS. Outcomes in patients with COVID-19 infection taking ACEI/ARB. Curr Cardiol Rep 2020; 22: 31.

7 Imai Y, Kuba K, Penninger JM. The discovery of angiotensin-converting enzyme 2 and its role in acute lung injury in mice. Exp Physiol 2008; 93: 543-548.

8 Schouten LR, van Kaam AH, Kohse F, et al. Age-dependent differences in pulmonary host responses in ARDS: a prospective observational cohort study. Ann Intensive Care 2019; 9: 55.

9 Fernández-Atucha A, Izagirre A, Fraile-Bermúdez AB, et al. Sex differences in the aging pattern of renin-angiotensin system serum peptidases. Biol Sex Differ 2017; 8: 5.

\section{(1) CrossMark}

From the authors:

We thank G.J. Porter for his comments on our recently published editorial: "Will children reveal their secret? The coronavirus dilemma" [1]. In the editorial, we reviewed some of the strongest evidence that may support our perspective. It was beyond the purpose of our manuscript to provide a full description of the renin-angiotensin-aldosterone system (RAAS) and angiotensin-converting enzyme 2 (ACE2) receptor. We strongly agree that evidence about the role of ACE2 in severe acute respiratory syndrome coronavirus

@ERSpublications

The role of ACE2 receptor in SARS-CoV-2 infection and in COVID-19 outcomes is still debated, especially in children https://bit.ly/35ZLf7V

Cite this article as: Midulla F, Cristiani L, Mancino E, et al. Will children reveal their secret? The coronavirus dilemma. Eur Respir J 2020; 55: 2001617 [https://doi.org/10.1183/13993003.01617-2020]. 
2 (SARS-CoV-2) infection is conflicting and our putative perspective was clearly pointed out in the paper. The debate around the role of ACE2 in SARS-CoV-2 infection is ongoing and we appreciate the chance that G.J. Porter has given us to better elucidate some of its main aspects.

Robust evidence against all of the body of literature about ACE2 downregulation in chronic conditions was recently provided by LEUNG et al. [2], who demonstrated increased ACE2 expression in lung biopsies of current smokers and in patients with COPD. Despite not investigating the association between their findings and the risk of SARS-CoV-2 infection, they suggested that ACE2 upregulation could partially explain the increased risk of SARS-CoV-2 infection in these subpopulations.

In his letter, G.J. Porter also stresses the uncertainty about the renin-angiotensin system and ACE2 derangement during SARS-CoV-2 infection in children, and their variability under physiological and pathological conditions, providing evidence that we would like to discuss further. To our knowledge, ACE2 serum levels were not investigated in the study by Liu et al. [3]. On the contrary, plasma concentrations of angiotensin II were measured, resulting in a markedly higher concentration of its plasma levels in patients with coronavirus disease 2019 (COVID-19) than in healthy controls. These findings are in accordance with our hypothesis, that ACE2 dysregulation and angiotensin II elevated levels could lead to inflammation and lung injury.

ACE2 age-related expression was also questioned. We agree with G.J. Porter that the results of the preclinical studies about ACE2 age-related expression in rat models cannot be fully translated to humans. In a report by FERNÁNDEZ-ATUCHA et al. [4], 118 healthy individuals, ranging 41-70 years old, were enrolled and serum ACE2 activity was measured. Results showed significantly higher ACE2 activity in older women and no differences in men. However, in that study, children were not investigated. In addition, concerns about serum ACE2 activity measurement have recently been highlighted, as it may not be a reliable indicator of the membrane-bound form [5, 6]. Regarding ACE2 age-related variability, SCHOUTEN et al. [7] reported no significant difference in lung ACE2 activity among patients of all ages with acute respiratory distress syndrome. However, as the authors state, the results might have been underpowered due to the relatively small sample size of each age group. Moreover, dilution differences of bronchoalveolar lavage return fluid could have influenced the biomarkers' final concentration. Notably, ACE2 levels were only measured in the alveolar compartment, which does not reflect the pathological response of the whole RAAS.

Finally, VADUGanathan et al. [5] recently discussed the role of RAAS inhibitors in patients with COVID-19. The study had a clear focus on their protective rather than detrimental effect in SARS-CoV-2 infection, and highlighted that the hypothesis regarding the beneficial role of ACE2 has led to recombinant ACE2 protein administration trials in order to prevent organ injury (ClinicalTrials.gov number, NCT04287686). Recent clinical trials have also offered us the opportunity to clarify that chronic use of angiotensin-II receptor blockers and ACE inhibitors (and thus, hypothetically, the lung-specific upregulation of ACE2) is not associated with an increased risk of COVID-19 or severe outcomes in COVID-19 [8, 9].

In conclusion, we agree with G.J. Porter that the role of ACE2 receptor in SARS-CoV-2 infection and in COVID-19 outcomes is still being debated, especially in children. Data about lung-specific ACE2 expression in healthy children and in those with COVID-19 are lacking. Further studies about the interconnection of RAAS and SARS-CoV-2 infection are needed, especially in the paediatric age group, in order for children to reveal their hidden secret.

Fabio Midulla, Luca Cristiani and Enrica Mancino

Dept of Maternal Science, Paediatric Emergency Service, Sapienza University of Rome, Rome, Italy.

Correspondence: Fabio Midulla, University of Rome, Cystic Fibrosis, V.le Regina Elena, 324, Rome 00161, Italy. E-mail: midulla@uniromal.it

Received: 6 May 2020 | Accepted: 7 May 2020

Conflict of interest: None declared.

References

1 Cristiani L, Mancino E, Matera L, et al. Will children reveal their secret? The coronavirus dilemma. Eur Respir J 2020; 55: 2000749.

2 Leung JM, Yang CX, Tam A, et al. ACE-2 expression in the small airway epithelia of smokers and COPD patients: implications for COVID-19. Eur Respir J 2020; 55: 2000688.

3 Liu Y, Yang Y, Zhang C, et al. Clinical and biochemical indexes from 2019-nCoV infected patients linked to viral loads and lung injury. Sci China Life Sci 2020; 63: 364-374. 
4 Fernández-Atucha A, Izagirre A, Fraile-Bermúdez AB, et al. Sex differences in the aging pattern of renin-angiotensin system serum peptidases. Biol Sex Differ 2017; 8: 5.

5 Vaduganathan M, Vardeny O, Michel T, et al. Renin-angiotensin-aldosterone system inhibitors in patients with COVID-19. N Engl J Med 2020; 382: 1653-1659.

6 Lambert DW, Yarski M, Warner FJ, et al. Tumor necrosis factor- $\alpha$ convertase (ADAM17) mediates regulated ectodomain shedding of the severe-acute respiratory syndrome-coronavirus (SARS-CoV) receptor, angiotensin-converting enzyme-2 (ACE2). J Biol Chem 2005; 280: 30113-30119.

7 Schouten LR, van Kaam AH, Kohse F, et al. Age-dependent differences in pulmonary host responses in ARDS: a prospective observational cohort study. Ann Intensive Care 2019; 9: 55.

8 Reynolds HR, Adhikari S, Pulgarin C, et al. Renin-angiotensin-aldosterone system inhibitors and risk of COVID-19. N Engl J Med 2020; in press [https://doi.org/ 10.1056/NEJMoa2008975].

9 Mancia G, Rea F, Ludergnani M, et al. Renin-angiotensin-aldosterone system blockers and the risk of COVID-19. N Engl J Med 2020; in press [https://doi.org/10.1056/NEJMoa2006923]. 